\title{
New vaccines - which ones should we choose?
}

\author{
Joan Robinson MD
}

$\mathrm{T}$ hanks to the British North America Act of 1867, responsibility for health care belongs to the provinces and territories. Thus, we have 13 different immunization schedules across Canada. The variation in the routine schedules is based on different personal opinions and priorities of individuals making the decisions on the introduction of new vaccines, different interpretations of short-term and long-term benefits and costs, different degrees of public pressure, different epidemiology and different sizes of caches available to pay for new vaccines.

The quadrivalent conjugated meningococcal vaccine, human papilloma virus (HPV) vaccine for females and rotavirus vaccine were all licensed at approximately the same time in Canada, and there are currently two brands of each vaccine available. The HPV vaccine has subsequently been licensed for use in males. All of these vaccines appear to have high efficacy in the short term, although concerns about waning titres from the quadrivalent conjugated meningococcal vaccine have resulted in the introduction of a routine second dose for adolescents in the United States (1). All of these vaccines have excellent safety profiles; it appears that both currently licensed rotavirus vaccines cause intussusception in approximately one in 100,000 children, but vaccine benefits clearly outweigh this tiny risk (2).

The personal opinions of individuals entrusted to make decisions concerning routine use of vaccines are a powerful force. There is increasing recognition that cancers beyond the cervix are potentially preventable using the HPV vaccine (3), but 'experts' would all have different thresholds for how many cancers need to be prevented decades down the road to warrant a routine program. Data from the United States National Health and Nutrition Examination Survey (NHANES) revealed that, between 1999 and 2004, 5.6\% (95\% CI $4.9 \%$ to $6.4 \%$ ) of sexually active individuals 15 to 59 years of age had self-reported genital warts in the past (4). Canadian data would likely be similar. Some would consider this burden of disease alone to be an indication that both sexes should receive the HPV vaccine, while others would argue that genital warts are usually benign, or that working harder at increasing the immunization rates in females is a more cost-effective way to prevent all HPV disease. Personal opinion also plays a role in decisions concerning rotavirus vaccines. Engendering enthusiasm for this vaccine among clinicians has been challenging because many consider the benefits to be minimal. However, it has become evident that the limited availability of diagnostics has led to under-recognition of the burden that diarrhea from rotavirus places on parents and on the health care system (5).

Public opinion must be considered if we are to maintain public confidence in immunization programs. Although many children with meningococcemia recover with no sequelae, we all know that parents would pay any price to prevent purpura fulminans. Therefore, although infections with the serotypes contained in the quadrivalent conjugated vaccine (A, C, Y and W135) are rare, adolescent immunization programs with this vaccine are emerging across the country. Licensure of a meningococcal B vaccine appears to be imminent in Canada, which will result in yet one more difficult decision for each jurisdiction, complicated by the fact that because this vaccine is not based on the polysaccharide capsule, a correlate of protection is not established and estimates of efficacy are tenuous (6).

The high costs of vaccine development are compounded by the tremendous risks that manufacturers take now that regulatory agencies are likely to reject a product if there is a potential link to serious adverse events in the prelicensure stage, as recently occurred with motavizumab, a high-titre monoclonal antibody for respiratory syncytial virus (7). During the 1980s, the cost of hemodialysis was approximately $\$ 50,000$ per patient per year. This figure was later informally accepted as the cost that society should be willing to pay if it would provide a person with one extra year of reasonably highquality life (otherwise known as quality-adjusted life years [QALY]). Although all would agree that quality of life is extremely subjective, 0.5 QALYs has been used to refer to one extra year of life at one-half the normal quality because of disability or discomfort. By examining the cost of adverse events resulting from vaccines and the costs saved by disease averted, cost-utility analysis has been used to determine the potential cost per QALY gained by routine use of new vaccines. Comparison of the values obtained for different vaccines should be a vital piece of information in choosing one vaccine over another. The difficulty is that many of the factors that are included in cost-utility equations are unknown. For example, long-term effectiveness of the vaccine may change. Replacement disease (ie, when nonvaccine serotypes or strains become more prevalent, as occurred to some degree with 7-valent pneumococcal conjugated vaccine) may decrease the efficacy over time. On the other hand, herd immunity may be greater than predicted (which also occurred with the 7-valent pneumococcal conjugated vaccine), such that benefits from the vaccine may extend beyond those who are vaccinated. Another example of the difficulty in choosing factors to use in cost-utility equations is that, as indicated earlier, determining the QALY to assign to a year when one has genital warts or is undergoing treatment for cancer is far from an exact science.

Even if we were to develop a uniform and fair way to assign cost per QALY gained by routine use of new vaccines, the problem remains as to what amount society can afford to pay. As the number of potentially life-prolonging - but extremely costly - technologies expands, it may no longer be practical for the health care system to pay $\$ 50,000$ per QALY that we could gain by implementing all of these technologies.

Although it should not be front and centre in the decisions made about vaccines, we need to recognize that when novel vaccines developed for routine use are not approved for such, they may disappear from the market (as happened with the vaccine for Lyme disease) (8). These decisions against widespread use of vaccines are clear disincentives for the vaccine industry to continue to develop new vaccines.

It is inefficient to have 13 jurisdictions making the same complex decisions about immunization schedules (9). Perhaps at some magical point in the future, we can over-rule the British North America Act and insist on a single schedule, with allowances made for exceptions when local epidemiology is unique. 


\section{REFERENCES}

1. Cohn AC, MacNeil JR, Clark TA, et al. Centers for Disease Control and Prevention (CDC). Prevention and control of meningococcal disease: Recommendations of the Advisory Committee on Immunization Practices (ACIP). MMWR Recomm Rep 2013;62(RR-2):1-28.

2. Parashar UD, Orenstein WA. Intussusception and rotavirus vaccination - Balancing risk against benefit. Clin Infect Dis 2013; August 19 [Epub ahead of print].

3. Grulich AE, Jin F, Conway EL, Stein AN, Hocking J. Cancers attributable to human papillomavirus infection. Sex Health 2010;7:244-52.

4. Dinh TH, Sternberg M, Dunne EF, Markowitz LE. Genital warts among 18- to 59-year-olds in the United States, National Health and Nutrition Examination Survey, 1999-2004. Sex Transm Dis 2008;35:357-60.

5. Bettinger JA, Wills K, Le Saux N, Scheifele DW, Halperin SA, Vaudry W. Heterogeneity of rotavirus testing and admitting practices for gastroenteritis among 12 tertiary care pediatric hospitals: Implications for surveillance. Can J Infect Dis Med Microbiol 2011;22:15-8.
6. Bettinger JA, Scheifele DW, Halperin SA, et al; for the members of the Canadian Immunization Monitoring Active (IMPACT)

Program. Diversity of Canadian meningococcal serogroup B isolates and estimated coverage by an investigational meningococcal serogroup B vaccine (4CMenB). Vaccine 2013; April 12 (Epub online ahead of print).

7. AstraZeneca discontinues development of motavizumab for RSV prophylaxis indication. <www.astrazeneca.com/Media/Press-releases/ Article/AstraZeneca-discontinues-motavizumab-RSV> (Accessed October 1, 2013).

8. Plotkin SA. Correcting a public health fiasco: The need for a new vaccine against Lyme disease. Clin Infect Dis 2011;52(Suppl 3):S271-5.

9. MacDonald NE, Bortolussi R; Canadian Paediatric Society, Infectious Diseases and Immunization Committee. A harmonized immunization system for Canada: A call to action. Paediatr Child Health 2011;16:29-31. 


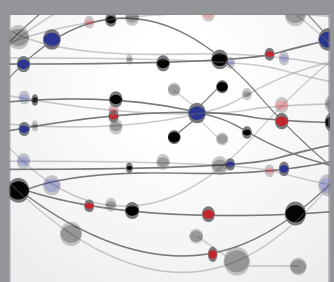

The Scientific World Journal
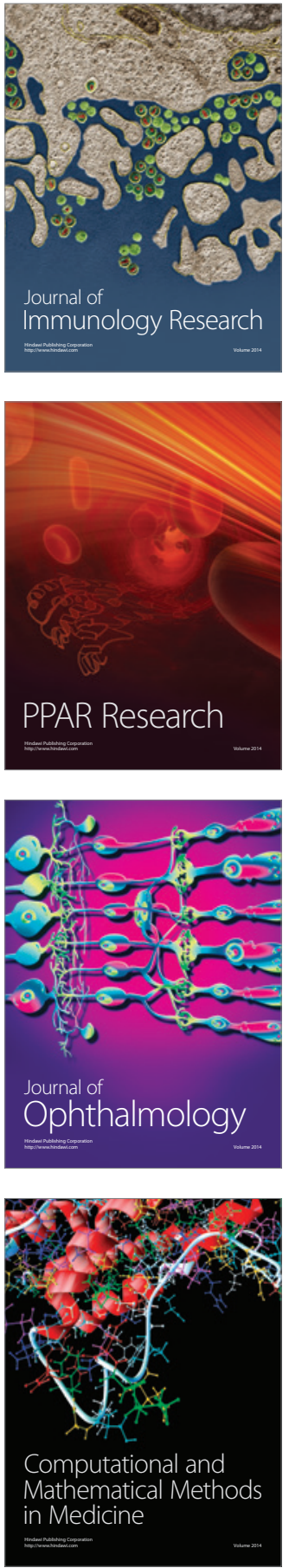

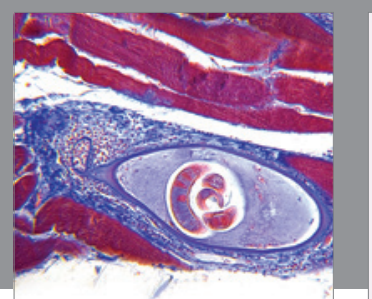

Gastroenterology Research and Practice

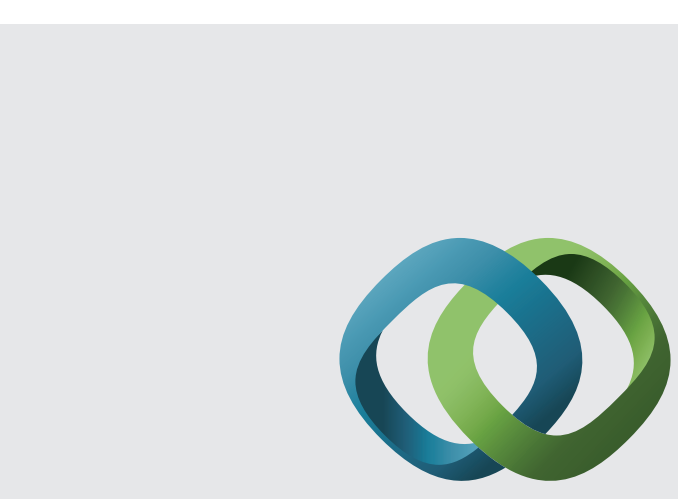

\section{Hindawi}

Submit your manuscripts at

http://www.hindawi.com
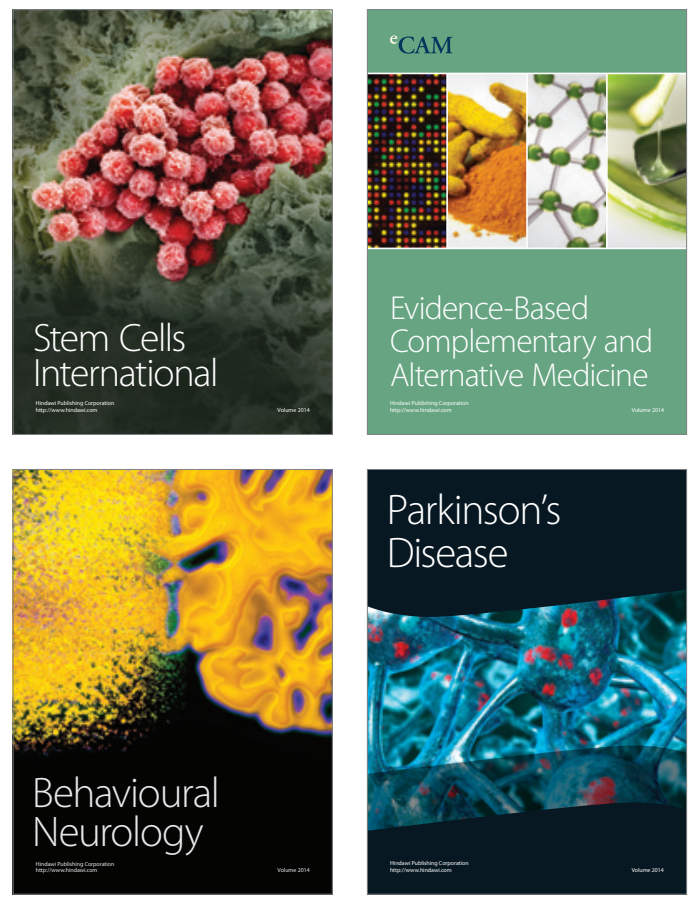
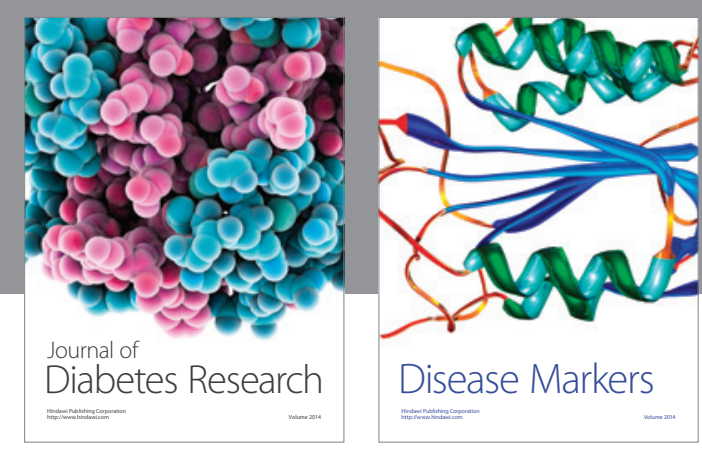

Disease Markers
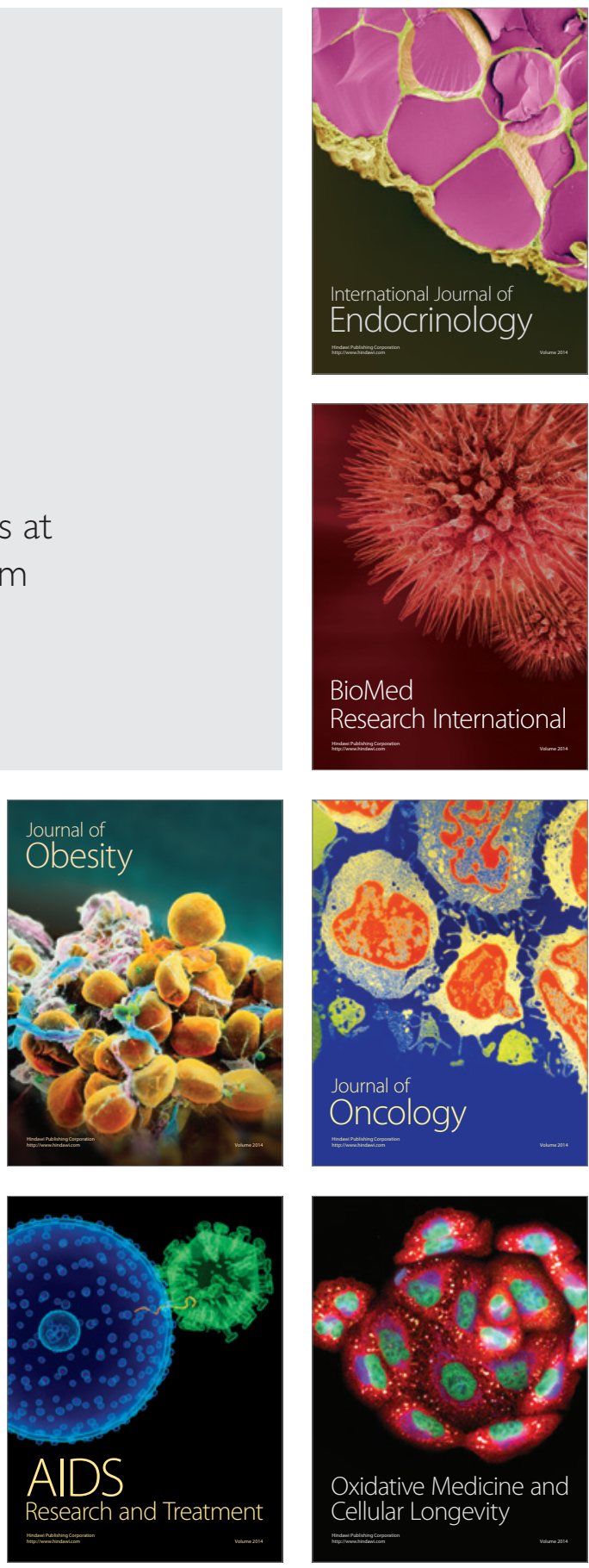